\title{
Review
}

Ophthalmologica

\section{Neurotrophic Keratitis}

\author{
F. Semeraro ${ }^{a}$ E. Forbice ${ }^{a}$ V. Romano ${ }^{b}$ M. Angi ${ }^{b} \quad$ M.R. Romano $o^{b, c}$ \\ M.E. Filippellib R. Di lorio ${ }^{b} \quad$ C. Costagliolab
}

${ }^{a}$ Eye Clinic, Department of Ophthalmology, University of Brescia, Brescia, ${ }^{b}$ Eye Clinic, Department of Health

Sciences, University of Molise, Campobasso, and ${ }^{\mathrm{C} E y e}$ Unit, Istituto Clinico Humanitas, Rozzano, Italy

\section{Key Words}

Neurotrophic keratitis · Corneal sensitivity · Trigeminal

nerve $\cdot$ Corneal vascularization $\cdot$ Stromal ulcers

\begin{abstract}
Neurotrophic keratitis (NK) is a rare degenerative corneal disease that occurs as a result of partial or total impairment of trigeminal innervations, leading to a reduction (hypoesthesia) in or loss (anaesthesia) of corneal sensitivity. The impairment of sensory innervation causes a reduction in the lacrimation reflex and the vitality, metabolism and mitosis of epithelial cells, with subsequent deficiency in epithelial repair, stromal and intracellular oedema, loss of microvilli, and abnormal development of the basal lamina. Several recent studies have proposed different therapies based on different aetiopathogenetic theories. The aim of the therapy is to treat aetiopathogenesis and, at the same time, promote corneal healing. In this paper, we report the aetiology, diagnosis, management, and medical and surgical treatment of NK, also indicating future treatments based on the most recent studies.

○) 2013 S. Karger AG, Basel
\end{abstract}

\section{Introduction}

Neurotrophic keratitis (NK) is a degenerative corneal disease that follows a partial or total impairment of trigeminal innervation, leading to a reduction (hypoesthe- sia) in or loss (anaesthesia) of corneal sensitivity [1]. In this article, we review the aetiology, diagnosis and management of NK.

\section{Aetiopathogenesis}

An animal study conducted in 1954 revealed the molecular basis of this disease for the first time, showing its development by sectioning of the trigeminal nerve [2]. The trigeminal ophthalmic branch has 2 reflex arcs: one motor arc, regulating the opening and closing of the eyelids, and one autonomic arc, regulating the lacrimal gland, Meibomian gland and goblet cell secretion. The integration of these 2 reflex arcs ensures the stability of the tear film and, together with related neurotrophic factors (neurotrophins), the vitality of the epithelium and stroma [3]. Cavanagh et al. $[4,5]$ described the bi-directional regulation of corneal epithelial proliferation through sensory and autonomic sympathetic innervation. The impairment of sensory innervation, as demonstrated by Sigelman [2], causes a reduction in the lacrimation reflex and the vitality, metabolism and mitosis of epithelial cells, with subsequent deficiency in epithelial repair, stromal and intracellular oedema, loss of microvilli, and abnormal development of the basal lamina $[2,6-8]$.

The study of NK has directed the attention of researchers to the role of neurotransmitters such as substance $\mathrm{P}$ (SP), calcitonin gene-related peptide, neuropeptide $\mathrm{Y}$, vasoactive intestinal peptide, galanin, methionine-enkeph-

\section{KARGER}

E-Mail karger@karger.com

www.karger.com/oph
(C) 2013 S. Karger AG, Basel

0030-3755/13/2314-0191\$38.00/0
Eliana Forbice

Eye Clinic, Department of Ophthalmology, University of Brescia

Piazzale Spedali Civili 1

IT-25100 Brescia (Italy)

E-Mail elianaforbice@ email.it 
alin, catecholamine, and acetylcholine (Ach) $[5,9]$. Studies have shown a decrease in SP and Ach resulting from injury to the corneal nerves $[10,11]$, the ability of SP alone to stimulate the synthesis and growth of corneal epithelial cells [12], and epithelial proliferation in vitro by SP, cholecystokinin gene-related peptide and Ach $[5,12,13]$. Although studies performed in humans could not show that the treatment with SP alone promotes corneal reepithelialization [14], Chikama et al. [15] reported the complete recovery of a patient with NK after treatment with SP and insulin-like growth factor-1 (IGF-1) eye drops. These studies have suggested that treatment with a combination of SP and IGF-1 stimulates epithelial cell migration, integrin $\beta$ and $\alpha_{5}$, essential for epithelial cell anchoring. Ach released from sensory neurons increases intranuclear cyclic guanosine monophosphate (cGMP) levels and promotes epithelial growth, and the severe depletion of Ach seen in corneal denervation causes decreased epithelial growth. Adrenergic stimulation partially reverses the effects of sympathetic denervation $[11,16$, 17], and in animal studies, in which corneal anaesthesia is induced, corneal epithelial effects are mitigated by cervical sympathetic denervation [11].

The role of neurotrophins such as nerve growth factor (NGF) in NK has also generated increasing interest [18, 19]. These neurotrophins seem essential for the maintenance and regulatory functions of the nervous system. The discovery of the first neurotrophin, NGF, triggered the search for factors influencing the development and maintenance of the nervous system [20]. NGF is a neurotrophin essential to the development and survival of selected neurons, including sympathetic and sensory neurons, and for trophic support after neuronal injuries. It is normally present in the healthy cornea, where it regulates the proliferation and differentiation of epithelial cells. NGF also appears to be involved in epithelial and stromal interactions that induce stromal healing as well as in the remodeling mechanism that promotes the onset of stromal opacity.

NK can be the expression of systemic or ocular congenital or iatrogenic diseases resulting from damage to the fifth cranial nerve (table 1). The most common causes of loss of corneal sensitivity are herpes keratitis, chemical burns, long-term use of contact lenses, corneal surgery [21], ablative procedures for trigeminal neuralgia [22], and surgical procedures for reduction of jaw fractures [23]. Other less frequent causes are space-occupying intracranial masses (e.g., schwannoma, meningioma and aneurysms) that can lead to compression of the nerve and reduce corneal sensitivity. Systemic diseases that may compromise trigeminal function are diabetes, multiple
Table 1. Aetiopathogenesis of NK

\begin{tabular}{ll}
\hline Infections & Herpes simplex \\
& Herpes zoster \\
& Leprosy \\
\hline Corneal pathologies & Chemical burns \\
& Contact lens wear \\
& Surgeries \\
& Laser-assisted in situ keratomileusis \\
& Corneal incision \\
& Lamellar and penetrating keratoplasty \\
& Dystrophies \\
& Lattice \\
& Granular \\
\hline Topical medications & Anaesthetics \\
& Timolol \\
& Betaxolol \\
& Trifluridine \\
& Sulfacetamide \\
\hline Cranial nerve V palsy & Trigeminal neuralgia surgery \\
& Neoplasm \\
& Aneurysm \\
& Facial trauma \\
& Congenital \\
& Riley-Day syndrome \\
& Möbius corneal hypoesthesia \\
\hline Miscellaneous & Diabetes \\
& Vitamin A deficiency \\
& Multiple sclerosis \\
& Increasing age \\
& Adie's syndrome \\
\hline & \\
& \\
& \\
& \\
&
\end{tabular}

sclerosis and leprosy $[24,25]$. NK may also be a complication of radiation therapy. Congenital causes, such as the Ridley-Day syndrome, anhidrotic ectodermal dysplasia, Moebius syndrome, Goldenhar syndrome, and congenital corneal anaesthesia, are very rare.

\section{Diagnosis}

Epidemiological data on NK have not been reported in the literature. Diagnosis can be achieved by obtaining a detailed medical history to investigate all possible risk factors, followed by a careful examination of the ocular and periocular area, including the globe and ocular adnexa.

\section{Medical History}

Previous episodes of redness and eye pain or the presence of cutaneous blistering or scarring suggest previous herpetic infections. A history of corneal surgery, trauma, 
abuse of topical anaesthetics, long-term use of topical medications, chemical burns, or contact lens abuse may be contributory. Long-term use of eye drops such as timolol, betaxolol, sulfacetamide sodium, or diclofenac can cause a loss of corneal sensitivity, as can the abuse of topical anaesthetics $[3,26,27]$. Corneal hypoesthesia can sometimes occur in advanced stromal dystrophies such as latex or granular dystrophies [3]. Any neurological signs or symptoms or expression of fifth cranial nerve disease such as brain tumours, vascular accidents and thromboangiitis obliterans should be explored. A history of previous surgical resection of an acoustic neuroma may suggest a iatrogenic trigeminal nerve injury. The presence of hearing problems may indicate intracranial tumours such as neuromas of the eighth cranial nerve, especially when the fifth and seventh cranial nerves are involved [28]. A history of long-term therapy with neuroleptics, antipsychotics and antihistamines should be investigated, as should a history of diabetes mellitus, because reduction in corneal sensitivity in diabetes increases with the duration of the disease [24].

\section{Physical Examination}

The symptoms reported by the patient vary depending on the degree of corneal anaesthesia, but usually, the patient complains of a red eye and a slight reduction in visual acuity. The corneal sensitivity test is the basic examination and can be performed by touching the central and peripheral cornea with a Cochet-Bonnet aesthesiometer, which locates and quantifies the loss of corneal sensitivity by recording the patient's response to the touch of a nylon thread (less than $5 \mathrm{~mm}$ is regarded as clinically significant corneal hyposensitivity) [29]; a less sensitive test is the response to touch with the tip of a cotton swab [30]. In general, the severity of NK is related to the severity of corneal sensory impairment. The disease is usually unilateral, but if it is bilateral, there will also be a reduction in blinking as well as reduced tear production due to lack of the afferent arm of the lacrimation reflex $[31,32]$. Fluorescein, rose bengal and lissamine green stains show a reduction in tear break-up time and formation of geographic dry spots and corneal and/or conjunctival epithelial defects. It is useful to perform a careful examination of the eyelid, including eyelid edges, positions and motility, to rule out exposure keratitis and diagnose blepharitis, which is often associated with NK. Microbiological examination of large, persistent epithelial defects must be performed to exclude bacterial, fungal or viral infection. The combination of an afferent pupillary defect and hypoesthesia must be studied to rule
Table 2. Classification of NK

\begin{tabular}{ll}
\hline Stage 1 & Punctate epithelial staining \\
& Decreased tear breakup test \\
& Rose bengal staining of inferior palpebral conjunctiva \\
& Dellen \\
& Gaule spots \\
& Stromal scarring \\
\hline Stage 2 & Epithelial defect \\
& Stromal swelling \\
& Surrounding rim of loose epithelium \\
& Rare anterior chamber reaction \\
\hline Stage 3 & Corneal ulcer \\
& Stromal lysis \\
& Perforation \\
\hline
\end{tabular}

out intraconal orbital nerve injury. A reduction in accommodation can indicate damage to the motor ciliary nerve of the ciliary ganglion. Iris atrophy is often an expression of a previous herpetic keratouveitis or a lepromatous lesion. NK should be suspected in all patients who have a discrepancy between reported symptoms and ocular signs or in patients who have a decreased frequency of eyelid closure.

\section{Classification of $\mathrm{NK}$}

NK can be classified into 3 stages according to the Mackie classification (table 2) [21,22]. The first stage is characterized by punctate keratitis, epithelial hyperplasia, stromal scarring, and corneal neovascularization. The second stage is characterized by a persistent epithelial defect $[21,22]$, usually in the paracentral area, of oval shape with the horizontal axis surrounded by an irregular, oedematous, opaque epithelium capable of spontaneous detachment. A reaction in the anterior chamber or sterile hypopyon is rarely seen. At this stage, the mechanism of loss of the corneal epithelium is similar to that of recurrent erosions, promoted by reduced lubrication of the corneal surface and by the abnormal corneal epithelium. In the third stage, there is stromal involvement that appears as a stromal corneal ulcer and stromal oedema and infiltrates; this may result in perforation and/or corneal thinning due to stromal melting [21,22].

\section{Differential Diagnosis}

Stage 1 NK may be confused with other eye diseases such as dry eye, exposure keratitis, topical drug toxicity, contact lens abuse, and corneal limbal deficiency. The presence of marked symptoms of burning, foreign body sensation, photophobia, and dry eye may direct the diag- 
nosis towards other diseases of the ocular surface, but the hallmark of NK is anaesthesia. Sometimes, the epithelial defect can take on a dendritic form, but it can be distinguished from herpetic infection by the absence of benching. Limbal stem cell deficiency is distinguished by impression cytology, which enables the identification of cytokeratin (CK; the corneal epithelium is positive for CK3 and negative for CK19, while the conjunctival epithelium is positive for CK19 and negative for CK3) [33]. The clinical history, signs and symptoms, and results of microbiological tests for bacteria, fungi and viruses allow a definitive diagnosis. Any topical treatment should be discontinued to rule out iatrogenic corneal ulcers, and evaluation for systemic immune disorders should be considered.

\section{Treatment}

The aim of the therapy is to prevent the progression of corneal damage and promote healing of the corneal epithelium [34]. In stage 1 disease, all topical medications and systemic therapies such as neuroleptics, antipsychotics and antihistamines that can cause NK should be discontinued. The ocular surface can be preserved through the use of artificial tears without preservatives every $2-4 \mathrm{~h}$ and a lubricant ointment at bedtime. The goal at this stage is to improve the quality of the corneal epithelium, prevent an epithelial breakdown and preserve corneal transparency. Comorbidities such as exposure keratitis or limbal deficiency worsen the prognosis and should be treated before beginning NK symptoms.

The goal of treatment for Mackie stage $2 \mathrm{NK}$ is to avoid the development of a corneal ulcer and promote healing of the epithelial defect. In addition to the discontinuation of all topical medications and the instillation of artificial tears without preservatives, as in the previous stage, it is essential that the patient be monitored frequently. The presence of asymptomatic disease may enable rapid progression to corneal perforation without the patient noticing it. In these cases, prophylactic antibiotic eye drops can be administered, and where microbiological examination is positive, it is essential to block concurrent keratitis with an effective antibiotic therapy. Corneal or scleral contact lens therapy can be used to promote healing while paying attention to the increased risks of secondary infection and lens-related sterile hypopyon [35-37]. The function of the lens is to provide a fluid cushion for the cornea and protect it from the rubbing effects of the lids [38-40]. Some authors have no- ticed an improvement in the cornea through the use of temporary silicone plugs to help increase tear volume [41].

In the event of large epithelial defects or corneal ulcers that do not respond to treatment with artificial tears or contact lens treatment (Mackie stage 3), it is useful to stop all treatment except artificial tears and prescribe antibiotic prophylaxis, as in previous stages. The main goal of treatment at this stage of NK is to prevent corneal thinning and perforation, and tarsorrhaphy has traditionally been considered the treatment of choice because it can be easily performed and is widely used. Alternatives to tarsorrhaphy include injection of botulinum toxin A to induce temporary ptosis of the upper eyelid or the use of a conjunctival flap to cover the corneal surface, but this compromises aesthetics and visual function [42-45].

New surgical and medical alternatives have recently been introduced. The surgical treatments are transplantation of amniotic membranes (AMs), conjunctival flaps and sympathectomy, but the most recent research considers that medical therapy can restore the nerve damage that forms the basis of the pathology. These new medical treatments include autologous serum eye drops, umbilical cord serum eye drops and neurotrophin eye drops [46].

Autologous serum contains many growth factors and tear components, such as epidermal growth factor, vitamin A, transforming growth factor- $\beta$, fibroblast growth factors, platelet-derived growth factor, hepatocyte growth factor, fibronectin, and serum antiproteases such as $\alpha 2$ macroglobulin; all these substances are suitable for facilitating the proliferation, migration and differentiation of the ocular surface epithelium. These properties have made autologous serum eye drops an effective treatment for severe dry eye in Sjögren's syndrome, persistent epithelial defects, superior limbic keratoconjunctivitis, and recurrent corneal erosion $[18,25]$. Autologous serum also harbours neurotrophic substances such as SP, IGF-1 and NGF and can be useful for the restoration of ocular surface integrity in patients with NK. Matsumoto et al. [47] first noted the presence of neurotrophic substances in autologous serum eye drops and their usefulness for the restoration of ocular surface integrity in patients with $\mathrm{NK}$ [47-53].

Umbilical cord serum also contains essential tear components, growth factors and neurotrophic factors and can be used in many ocular surface diseases, including dry eye syndrome, persistent epithelial defect and NK [54].
Semeraro/Forbice/Romano/Angi/ Romano/Filippelli/Di Iorio/Costagliola 
Topical steroids and sympathectomy have been suggested for NK therapy, because adrenergic neurotransmitters and prostaglandins lead to an increase in cAMP and reduce corneal epithelial mitosis, whereas Ach causes an increase in cyclic guanosine monophosphate and stimulates the growth of the corneal epithelium. Topical steroids may reduce the activity of inflammatory mediators and are particularly useful in patients with chemical burns $[4,5]$. However, the same topical steroids can inhibit stromal healing and increase the risk of stromal melting and corneal perforation [55]. The presence of inflammation in conjunction with a neurotrophic corneal ulceration further complicates treatment because the inflammatory response, together with the absence of sensory innervation, inhibits corneal epithelial growth $[4,5,56]$. The use of topical corticosteroids is indicated in cases in which inflammation is the driving cause of the persistent defect. Although theoretically, the use of topical nonsteroidal agents blocks the influence of prostaglandins on corneal epithelial growth, their clinical use has not been shown to have a relevant trophic effect on wound healing [55]. Diclofenac sodium in particular should be avoided because of its tendency to cause corneal hypoesthesia with topical use [57]. In studies on rabbits, sympathectomy has conferred improvement after damage to sensory nerves [58]; however, sympathectomy after chemically induced sensory nerve damage in humans did not yield the same results $[59,60]$.

The AM, a thin, avascular membrane comprising the innermost layer of the foetal membranes, is composed of 3 layers: epithelium, basement membrane (BM) and stroma. Each layer has specific biological properties that can be applied in different corneal diseases. The epithelium provides growth factors and cytokines useful for maintaining an undifferentiated epithelial phenotype during culture of limbal stem cells. Collagen IV/VII, laminin $1 / 5$ and fibronectin of the BM improve epithelial cell migration and adhesion to basal cells, induce epithelial differentiation, including goblet cells in the conjunctiva, and prevent apoptosis. Transforming growth factor- $\beta$, anti-inflammatory and anti-angiogenic proteins, and protease inhibition factors of the stromal matrix can suppress corneal myofibroblasts, proliferation and differentiation of normal and pathological conjunctival fibroblasts (inhibition of cicatrization), and inhibit inflammation and neovascularization. In NK, the AM is used to support epithelial adhesion, growth and differentiation and to prolong the lifespan of the epithelial progenitor cells [61-64].

Neurotrophic Keratitis
Ortuno-Prado and Alio [65] reported that the use of a Tutopatch with platelet-rich plasma was advantageous in a patient with neurotrophic ulcer, suggesting the use of Tutopatch as an alternative to AM transplantation. However, other studies are required to verify its effect.

Therapy with topical reductase inhibitors in diabetic patients produced improvement in corneal sensitivity and corneal epithelial cell morphology [66].

Several recent studies have proposed the use of neuropeptides and growth factors for the treatment of NK. Topical treatment with SP and IGF-1 induced healing in 2 patients who had disease recurrence within 1 year of follow-up [15, 67]. Epidermal growth factor has been used in human clinical trials with conflicting results [68, 69]. Topical treatment with NGF represents a promising therapy in the prevention of onset and progression of NK. Both in vivo and in vitro studies have shown that NGF induces the recovery of sensory neurons and the production of Ach in the central nervous system and of SP in the peripheral nervous system [70]. NGF also plays an important role in the balance between sensory and sympathetic innervations, modulating their functions [71]. Bonini et al. [18] demonstrated the restoration of epithelial integrity and corneal sensitivity in patients with NK treated with NGF. Other recent studies have shown the ability of NGF to induce healing of corneal ulcers by modulating epithelial tropism $[19,72]$.

Some authors have suggested use of collagenase inhibitors such as $\mathrm{N}$-acetylcysteine, tetracycline or medroxyprogesterone for corneal thinning [73]. Small perforations can be treated with application of cyanoacrylate glue and a soft contact lens or bandage [74, 75], but lamellar or penetrating keratoplasty is necessary to treat large perforations [75]. The success rate of these corneal transplants is low because of poor wound healing and the persistent risk of corneal epithelial defects from anaesthesia. Reed et al. [76] suggest that partial tarsorrhaphy can improve the success rate of keratoplasty, although the severity of sensory impairment is not reported $[18,19,72,77$, $78]$.

\section{Prognosis}

The prognosis of NK depends on several factors, namely, the cause of impairment of corneal sensitivity, the degree of corneal hypoaesthesia and the presence of concomitant diseases of the ocular surface. Obviously, the more severe the corneal sensory impairment, the greater the likelihood of disease progression [79]. 


\section{Follow-Up}

Patients with Mackie stage $1 \mathrm{NK}$ should be monitored closely, with re-evaluation after 3-7 days. Patients with stage 2 disease should be evaluated every 1-2 days until significant improvement is seen, then every $3-5$ days until resolution; those with stage 3 disease should be checked every day or hospitalized until significant improvement is seen. If tarsorrhaphy was performed, the opening between the eyelid margins can be enlarged after a couple of weeks, but attention must be paid because premature opening of tarsorrhaphy can cause recurrent corneal epithelial breakdown. At each visit, it is useful to perform staining to evaluate the corneal surface, a corneal sensitivity test and a Schirmer test; the best corrected visual acuity should also be evaluated.

\section{Conclusion}

$\mathrm{NK}$ is a degenerative corneal disease that occurs as a result of partial or total impairment of trigeminal innervations, leading to a reduction in or loss of corneal sensitivity. The aim of therapy is to treat the aetiopathogenesis and, at the same time, to promote the healing of the corneal epithelium, preventing the progression of corneal damage. In order to asses a correct treatment, the microbiological examination of large, persistent epithelial defects must be performed to exclude bacterial, fungal or viral infection. The neurotransmitters play a key role in the potential treatment of NK. The topical administration of NGF has actually demonstrated the ocular surface healing and immune-modulating actions in patients with infective and non-infective diseases of the ocular surface.

\section{References}

1 Mackie IA (ed): Neuroparalytic (neurotroph- 11 Shimizu T, et al: Capsaicin-induced corneal ic) keratitis. Symposium on contact lenses: transactions of the New Orleans Academy of Ophthalmology. St Louis, Mosby, 1973.

$\checkmark 2$ Sigelman S, Friedenwald JS: Mitotic and wound-healing activities of the corneal epithelium: effect of sensory denervation. Arch Ophthalmol 1954;52:46.

3 Chang BH, Gross EB Jr: Neurotrophic keratitis; in Krachmer JH, Mannis MJ, Holland EJ (eds): Cornea. London, Elsevier, vol 1, 2011.

4 Cavanagh HD, Colley AM, Pihlaja DJ: Persistent corneal epithelial defects. Int Ophthalmol Clin 1979;19:197.

5 Cavanagh HD, Colley AM: The molecular basis of neurotrophic keratitis. Acta Ophthalmol Suppl 1989;192:115-134.

6 Simone S: De ricerche sul contenuto in acqua totale ed in azoto totale della cornea di coniglio in condizione di cheratite neuroparalitic sperimentale. Arch Ottalmol 1958;62:151.

7 Alper MG: The anesthetic eye: an investigation of changes in the anterior ocular segment of the monkey caused by interrupting the trigeminal nerve at various levels along its course. Trans Am Ophthalmol Soc 1975;73: 323-365.

8 Mackie IA: Role of the corneal nerves in destructive disease of the cornea. Trans Ophthalmol Soc UK 1978;93:343.

9 Nagano T, et al: Effects of substance $\mathrm{P}$ and IGF-1 in corneal epithelial barrier function and wound healing in a rat model of neurotrophic keratopathy. Invest Ophthalmol Vis Sci 2003;44:3810-3815.

10 Mishima S: The effects of the denervation and the stimulation of the sympathetic and trigeminal nerve on the mitotic rate of the corneal epithelium in the rabbit. Jpn J Ophthalmol 1957;1:65-73. lesions in mice and the effects of chemical sympathectomy. J Pharmacol Exp Ther 1987; 243:690-695.

12 Reid TW, et al: Stimulation of epithelial cell growth by the neuropeptide substance P. J Cell Biochem 1993;52:476-485.

13 Mikulec AA, Tanelian DL: CGRP increases the rate of corneal re-epithelization in an in vitro whole mount preparation. J Ocul Pharmacol Ter 1996;12:417-423.

14 Kingsley RE, Marffurt CF: Topical substance $\mathrm{P}$ and corneal epithelia wound closure in the rabbit. Invest Ophthalmol Vis Sci 1997;38 388-395.

15 Chikama T, et al: Treatment of neurotrophic keratopathy with substance-P-derived peptide (FGLM) and insulin growth factor 1. Lancet 1998;351:1783-1784.

16 Abelli L, Geppetti P, Maggi CA: Relative contribution of sympathetic and sensory nerves to thermal nociception and tissue trophism in rats. Neuroscience 1993;57:739.

17 Perez E, et al: Effects of chronic sympathetic stimulation on corneal wound healing. Invest Ophthalmol Vis Sci 1987;28:221-224.

18 Bonini S, et al: Topical treatment with nerve growth factor for neurotrophic keratitis. Ophthalmology 2000;107:1347-1352.

19 Lambiase A, et al: Topical treatment with nerve growth factor for corneal neurotrophic ulcers. N Engl J Med 1998;338:1174-1180.

20 Levi-Montalcini R: The nerve growth factor 35 years later. Science 1987;237:1154-1162.

21 Gross EB Jr: Neurotrophic keratitis; in Krachmer JH, Mannis MJ, Holland EJ (eds): Cornea, ed 3. St Louis, Mosby, vol 1, 1997, p 1340.

22 Mackie IA: Neuroparalytic keratitis; in Roy FH, Meyer SM, Fraunfelder F (eds): Current
Ocular Therapy. Philadelphia, WB Saunders, 1995, pp 452-454.

23 Lanigan DT, Romanchuk K, Olson CK: Ophthalmic complications associated with orthognathic surgery. J Oral Maxillofac Surg 1993;51:480-494.

24 Hyndiuk RA, et al: Neurotrophic corneal ulcers in diabetes mellitus. Arch Ophthalmol 1977;95:2193-2196.

25 Hyndiuk RA, Kazarian EL, Schultz RO, Seideman S: Neurotrophic corneal ulcers in diabetes mellitus. Arch Ophthalmol 1977;95:21932196.

26 Kahook MY, Ammar DA: In vitro toxicity of topical ocular prostaglandin analogs and preservatives on corneal epithelial cells. J Ocul Pharmacol Ther 2010;26:259-263.

-27 Ammar DA, Noecker RJ, Kahook MY: Effects of benzalkonium chloride-preserved, polyquad-preserved, and sofZia-preserved topical glaucoma medications on human ocular epithelial cells. Adv Ther 2010;27:837845 .

28 Miller NR: Walsh and Hoyt's Clinical NeuroOphthalmology. Baltimore, Williams and Wilkins, 1985.

29 Norn MS: Measurement of sensitivity; in Norn MS (ed): External Eye Diseases: Methods of Examination. Copenhagen, Munksgaard International, 1974.

30 Faulkner WJ, Varley GA: Corneal diagnostic technique; in Krachmer JH, Mannis MJ, Holland EJ (eds): Cornea. St Louis, Mosby, 1997, pp 275-281.

31 Heigle TJ, Pflugfelder SC: Aqueous tear production in patients with neurotrophic keratitis. Cornea 1996;15:135-138.

32 Wright P, Mackie IA: Mucus in the healthy and diseased eye. Trans Ophthalmol Soc UK 1977;97:1-7. 
- 33 Elder MJ, Hiscott P, Dart JK: Intermediate filament expression by normal and diseased human corneal epithelium. Hum Pathol 1997; 28:1348-1354.

34 Lambiase A, et al: Management of neurotrophic keratopathy. Curr Opin Ophthalmol 1999;10:270-276.

35 Gould HL: Treatment of neurotrophic keratitis with scleral contact lenses. Eye Ear Nose Throat Mon 1967;46:1406-1414.

36 Kent HD, et al: Microbial keratitis and corneal ulceration associated with therapeutic soft contact lenses. CLAO J 1990;16:49-52.

- 37 Pfister RR: Clinical measures to promote corneal epithelial healing. Acta Ophthalmol Suppl 1992;202:73-83.

38 Grey F, et al: Scleral contact lens management of bilateral exposure and neurotrophic keratopathy. Cont Lens Anterior Eye 2012;35: 288-291.

-39 Alipour F, Kheirkhah A, Jabarvand Behrouz M: Use of mini scleral contact lenses in moderate to severe dry eye. Cont Lens Anterior Eye 2012;35:272-276.

40 Romero-Rangel T, et al: Gas-permeable scleral contact lens therapy in ocular surface disease. Am J Ophthalmol 2000;130:25-32.

41 Tai MC, et al: The clinical efficacy of silicone punctal plug therapy. Cornea 2002;21:135139.

-42 Kirkness CM, et al: Botulinum toxin A-induced protective ptosis in corneal disease. Ophthalmology 1988;95:473-480.

43 McNeill JI, Oh YH: An improved palpebral spring for the management of paralytic lagophthalmos. Ophthalmology 1991;98:715719.

-44 Gundersen T, Pearlson HR: Conjunctival flaps for corneal diseases: their usefulness and complications. Trans Am Ophthalmol Soc 1969;67:78-95.

45 Lugo M, Arentsen JJ: Treatment of neurotrophic ulcers with conjunctival flaps. Am J Ophthalmol 1987;103:711-712.

46 Lee SH, Tseng SC: Amniotic membrane transplantation for persistent epithelial defects with ulceration. Am J Ophthalmol 1997; 123:303-312.

47 Matsumoto Y, et al: Autologous serum application in the treatment of neurotrophic keratopathy. Ophthalmology 2004;111:1115-1120.

48 Poon AC, et al: Autologous serum eyedrops for dry eyes and epithelial defects: clinical and in vitro toxicity studies. Br J Ophthalmol 2001;85:1188-1197.
49 Young AL, et al: The use of autologous serum tears in persistent epithelial defect. Eye 2004; 18:609-614.

50 Tsubota K, et al: Treatment of persistent epithelial defect by autologous serum application. Ophthalmology 1999;106:1984-1989.

51 Kojima T, et al: The effect of autologous serum eyedrops in the treatment of severe dry eye disease: a prospective randomized case-control study. Am J Ophthalmol 2005;139:242-246.

52 Goto E, et al: Treatment of superior limbic keratoconjunctivitis by application of autologous serum. Cornea 2001;20:807-810.

53 del Castillo JM, et al: Treatment of recurrent corneal erosions using autologous serum. Cornea 2002;21:781-783.

54 Yoon KC, et al: Application of umbilical cord serum eyedrops for the treatment of dry eye syndrome. Cornea 2006;25:268-272.

55 Hersh PS, et al: Topical nonsteroidal agents and corneal wound healing. Arch Ophthalmol 1990;108:577-583.

56 Cavanagh HD, et al: The pathogenesis and treatment of persistent epithelial defects. Trans Sect Ophthalmol Am Acad Ophthalmol Otolaryngol 1976;81:754-769.

57 Szerenyi K, et al: Decrease in normal human corneal sensitivity with topical diclofenac sodium. Am J Ophthalmol 1994;118:312-315.

58 Fujita S, Miyazono Y, Ohba N: Capsaicin-induced corneal changes associated with sensory denervation in neonatal rat. Jpn J Ophthalmol 1987;31:412-424.

59 Adams GG, Cullen JF: Neuroparalytic keratitis and the effect of cervical sympathectomy following operative procedures for trigeminal neuralgia. Scott Med J 1987;32:86-88.

60 Welch E, Geary J: Current status of thoracic dorsal sympathectomy. J Vasc Surg 1984;1: 202-214.

61 Meller D, Tseng SCG: Conjunctival epithelial cell differentiation on amniotic membrane. Invest Ophthalmol Vis Sci 1999;40:878-886.

62 Meller D, Pires RT, Tseng SC: Ex vivo preservation and expansion of human limbal epithelial progenitor cells by amniotic membrane. Br J Ophthalmol 2002;86:463-471.

63 Chen HJ, Pires RT, Tseng SC: Amniotic membrane transplantation for severe neurotrophic corneal ulcers. Br J Ophthalmol 2000;84:826833.

64 Dua HS, et al: The amniotic membrane in ophthalmology. Surv Ophthalmol 2004;49: 51-77.

65 Ortuno-Prado VJ, Alio JL: Tratamiento de úlcera corneal neurotrófica con plasma rico en plaquetas y Tutopatch ${ }^{\circledR}$. Arch Soc Esp Oftalmol 2011;86:121-123.
66 Hosotani H, Ohashi Y, Yamada M, Tsubota $\mathrm{K}$ : Reversal of abnormal corneal epithelial cell morphologic characteristics and reduced corneal sensitivity in diabetic patients by aldose reductase inhibitor, CT-112. Am J Ophthalmol 1995;119:288-294.

67 Brown SM, et al: Neurotrophic and anhydrotic keratopathy treated with substance $\mathrm{P}$ and insulin like growth factor 1 . Arch Ophthalmol 1997;115:926-927.

68 Daniele S, Gilbard JP, Schepens CL: Treatment of persistent epithelial defects in neurotrophic keratitis with epidermal growth factor: a preliminary open study. Graefes Arch Clin Exp Ophthalmol 1992;230:314-317.

-69 Kandarakis AS, Page C, Kaufman HE: The effect of epidermal growth factor on epithelial healing after penetrating keratoplasty in human eyes. Am J Ophthalmol 1984;98:411-415.

70 Donnerer J, Amann R, Schuligoi R, Skofitsch G: Complete recovery by nerve growth factor of neuropeptide content and function in capsaicin-impaired sensory neurons. Brain Res 1996;741:103-108.

71 Schicho R, Skofitsch G, Donnerer J: Regenerative effect of human recombinant NGF on capsaicin-lesioned sensory neurons in the adult rat. Brain Res 1999;815:60-69.

72 Lambiase A, et al: Nerve growth factor promotes corneal healing: structural, biochemi$\mathrm{cal}$, and molecular analyses of rat and human corneas. Invest Ophthalmol Vis Sci 2000;41: 1063-1069.

73 Davis EA, Dohlman CH: Neurotrophic keratitis. Int Ophthalmol Clin 2001;41:1-11.

74 Fogle JA, Kenyon KR, Foster CS: Tissue adhesive arrests stromal melting in the human cornea. Am J Ophthalmol 1980;89:795-802.

75 Hirst LW, Smiddy WE, Stark WJ: Corneal perforations: changing methods of treatment, 1960-1980. Ophthalmology 1982;89:630-634.

-76 Reed JW, Joyner SJ, Knauer WJ III: Penetrating keratoplasty for herpes zoster keratopathy. Am J Ophthalmol 1989;107:257-261.

77 Steinle JJ: Topical administration of adrenergic receptor pharmaceutics and nerve growth factor. Clin Ophthalmol 2010;21:605-610.

78 Li W, Sun X, Wang Z, Li R, Li L: The effect of nerve growth factor on differentiation of corneal limbal epithelial cells to conjunctival goblet cells in vitro. Mol Vis 2010;15:27392744.

79 Cobo LM: Corneal complications of herpes zoster ophthalmicus. Prevention and treatment. Cornea 1988;7:50-56. 